\title{
Intensity of a source of atomic hydrogen based on a hot capillary
}

K. G. Tschersich

Citation: Journal of Applied Physics 87, 2565 (2000);

View online: https://doi.org/10.1063/1.372220

View Table of Contents: http://aip.scitation.org/toc/jap/87/5

Published by the American Institute of Physics

\section{Articles you may be interested in}

Formation of an atomic hydrogen beam by a hot capillary

Journal of Applied Physics 84, 4065 (1998); 10.1063/1.368619

Design and characterization of a thermal hydrogen atom source

Journal of Applied Physics 104, 034908 (2008); 10.1063/1.2963956

Simple source of atomic hydrogen for ultrahigh vacuum applications

Journal of Vacuum Science \& Technology A: Vacuum, Surfaces, and Films 11, 458 (1998); 10.1116/1.578754

Quantitative characterization of a highly effective atomic hydrogen doser

Journal of Vacuum Science \& Technology A: Vacuum, Surfaces, and Films 16, 2979 (1998); 10.1116/1.581449

Novel method for absolute quantification of the flux and angular distribution of a radical source for atomic hydrogen

Journal of Vacuum Science \& Technology A: Vacuum, Surfaces, and Films 18, 995 (2000); 10.1116/1.582289

Binding States of Hydrogen on Tungsten

The Journal of Chemical Physics 54, 4775 (2003); 10.1063/1.1674753

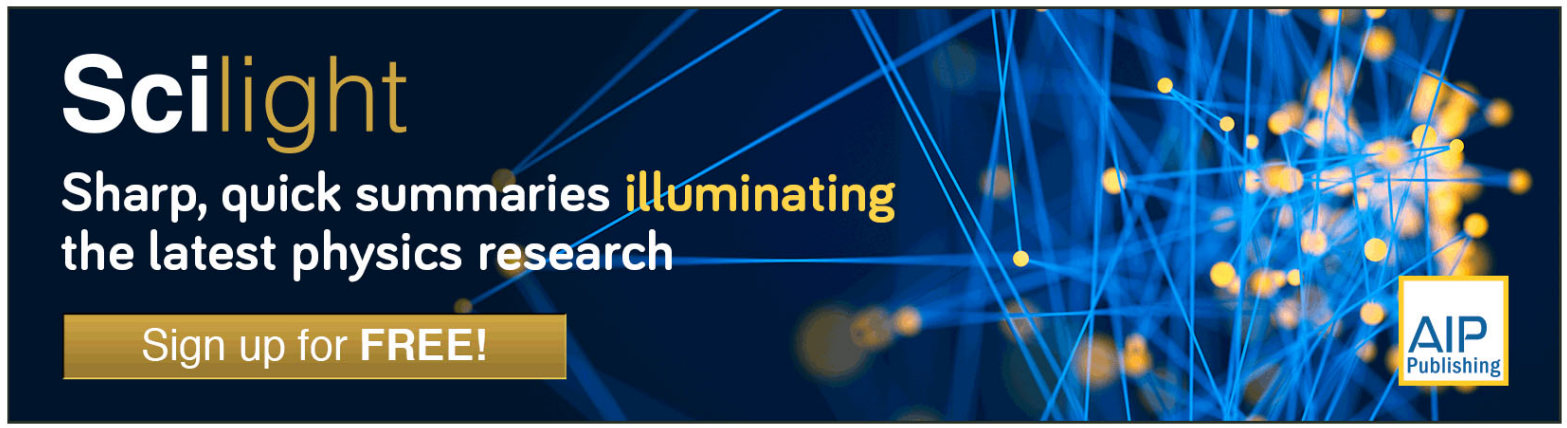




\title{
Intensity of a source of atomic hydrogen based on a hot capillary
}

\author{
K. G. Tschersich ${ }^{\mathrm{a})}$ \\ Institut für Grenzflächenforschung und Vakuumphysik, Forschungszentrum Jülich, D-52425 Jülich, Germany
}

(Received 21 September 1999; accepted for publication 3 November 1999)

\begin{abstract}
A source of atomic hydrogen incorporating a hot capillary was fabricated and investigated. The absolute and angularly resolved intensity of atomic hydrogen is derived from three experimentally determined quantities: (1) the flow rate of the hydrogen feed gas, (2) the degree of dissociation, and (3) the angular distribution of the emitted hydrogen atoms. The measurement of quantities (1) and (2) is reported here, quantity (3) has been investigated previously. The intensity of atomic hydrogen depends on the temperature of the capillary and the gas flow rate. At a temperature of $2600 \mathrm{~K}$ and a flow rate of $8 \times 10^{-3} \mathrm{mbar} \ell / \mathrm{s}$ the center-line intensity is about $4 \times 10^{17}$ atoms $/ \mathrm{sr}$ s. This is about four times the center-line intensity from a thin-walled aperture subjected to the same total flux. The enhancement is due to beam formation by the capillary and is even more pronounced at lower flow rates. (C) 2000 American Institute of Physics. [S0021-8979(00)03404-6]
\end{abstract}

\section{INTRODUCTION}

Atomic hydrogen is of considerable interest in semiconductor materials science and technology. ${ }^{1-3}$ In experimental work, atomic hydrogen is most often produced by thermal dissociation or in a plasma generated by radio-frequency radiation, microwave radiation, by electron cyclotron resonance, or by an arc discharge. We restrict ourselves here to sources equipped with some kind of opening from which the atomic hydrogen emanates. The hot filament type of source run in an ambient hydrogen atmosphere is not considered here.

Examining experimental papers one rarely finds the exposure to atomic hydrogen quantified by the flux density. Exposures are often characterized instead by the time of the exposure, by the number of Langmuirs quantifying the coincident exposure to molecular hydrogen, or by the electrical power sustaining a plasma. This illustrates the shortage of quantitative data on atomic hydrogen sources.

Absolute measurements of the intensity of atomic hydrogen sources can proceed along two different routes. In the first, an action of the atomic hydrogen is recorded. Sutoh et $a l .{ }^{4}$ measured the temperature increase of a platinum wire detector exposed to a thermal source constructed in-house ${ }^{5}$ as well as a commercial thermal source. ${ }^{6}$ The temperature increase was converted into a flux of atomic hydrogen via the heat of recombination. This conversion has to incorporate the coefficients of recombination as well as of accommodation. ${ }^{7}$ Samano et al. ${ }^{8}$ measured the temperature increase of a calorimetric sensor in order to determine the flux from an arc-based source. In addition to the heat of recombination they also considered the kinetic energy transferred to the sensor by the hyperthermal hydrogen atoms. Horn-von Hoegen and Golla ${ }^{9}$ observed the transformation of the $\operatorname{Si}(7 \times 7)$ reconstruction towards the $\operatorname{Si}(1 \times 1)$ structure and deduced the flux density from the time constant of this

a) Author to whom correspondence should be addressed; electronic mail: k.g.tschersich@fz-juelich.de transformation. All these methods rely on detailed knowledge of the action of atomic hydrogen and of the respective coefficients that enter into the conversion of the signal into a flux of atomic hydrogen.

The second and alternative route to absolute intensity data requires the simultaneous determination of three quantities: the flow rate of hydrogen gas to the source, the degree of dissociation in the source, and the angular distribution of the hydrogen atoms emanating from the source. Flow rate and degree of dissociation combine to give the total flux of atomic hydrogen. The angular distribution determines how much of that total flux is contained in a specific solid angle, e.g., in the one subtended by the surface of a sample to be exposed to atomic hydrogen.

Several authors measured the drop of the $\mathrm{H}_{2}$ signal of a quadrupole mass analyzer (QMA) induced by starting a radio-frequency source, ${ }^{10,11}$ a microwave source, ${ }^{11-13}$ or a thermal source. ${ }^{14}$ From the drop of the $\mathrm{H}_{2}$ signal they determined the fraction of dissociated hydrogen and (expect for Ref. 14). The total flux of atomic hydrogen. Fite et al. ${ }^{15}$ derived the fraction of dissociation from the currents of atomic and molecular ions produced by electron impact and from the known ratio of the respective cross sections. The dissociation fraction was furthermore determined by inelastic electron scattering. ${ }^{16,17}$

Van Zyl and Gealy ${ }^{18}$ fabricated and characterized a thermal source, the essential part of which was a hot capillary. By means of a differentially pumped QMA they measured the dissociation fraction and also reported the angular distribution of molecular hydrogen which was measured by pivoting the source about its opening.

A similar source was constructed by Bischler and Bertel $^{19}$ and quite recently characterized by Eibl et al. ${ }^{20}$ The degree of dissociation was investigated by thermal desorption spectroscopy, resonantly enhanced multiphoton ionization, and the pressure drop method dating back to Langmuir. ${ }^{21}$ The last method was reported to provide the highest accuracy. The authors concluded that the condition of Knudsen effusion does not hold at the exit of the capillary. 


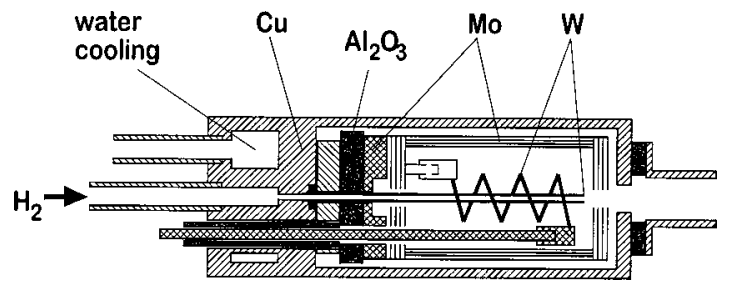

FIG. 1. Source of atomic hydrogen incorporating a capillary held by a water-cooled socket. The free end of the capillary is heated by electron impact and surrounded by radiation shields. Condenser plates at the front end of the source eliminate escaping electrons.

This had been assumed earlier in an attempt to estimate the degree of dissociation. ${ }^{19}$ The angular distribution of hydrogen molecules and atoms was investigated by Monte Carlo simulations which, in the case of hydrogen atoms, predicted an intensity dip on the capillary axis. Measurements of the angular distribution were made by adsorption of the hydrogen atoms on a gold foil and subsequent thermal desorption spectroscopy. The predicted dip could not be verified, which was attributed mainly to the moderate angular resolution determined by the size of the gold foil. ${ }^{20}$

None of the above-mentioned second route experiments provides the intensity of the respective source. Most often the intensity was simply inaccessible because the angular distribution was not investigated. It is the aim of the present article to pursue the second route up to the absolute intensity of atomic hydrogen.

In a previous article ${ }^{22}$ we described our source of atomic hydrogen which incorporates a hot capillary. We measured the angular distribution of the emitted hydrogen atoms by means of a QMA and considered the theory of beam formation due to molecular flow in the capillary. An analytic expression for the angular distribution of the atoms was derived and was used to fit the experimental data.

The present supplementary article reports on measurements of the flow rate of hydrogen gas to the source and of the degree of dissociation of the hydrogen emanating from the source. The result of the previous article is then included to evaluate the intensity. A particular feature of this procedure is that only the flow rate of hydrogen gas is determined in absolute numbers. The degree of dissociation and the angular distribution are measured as relative quantities. These three quantities will be treated in Sec. III A, III B, and III C and the performance of the source will be subsequently discussed.

\section{EXPERIMENTAL SETUP}

The source is described in our previous article ${ }^{22}$ and shown schematically in Fig. 1. Its core is a tungsten capillary which is heated by electron impact and surrounded by radiation shields. The capillary is fitted into a water cooled socket to which a gas feed line is attached. The temperature of the capillary orifice is measured by a disappearing filament optical pyrometer focused at the front end of the capillary. The reading of the pyrometer is corrected for the absorption of a glass window and the emissivity of tungsten. ${ }^{23,24}$ The mea-

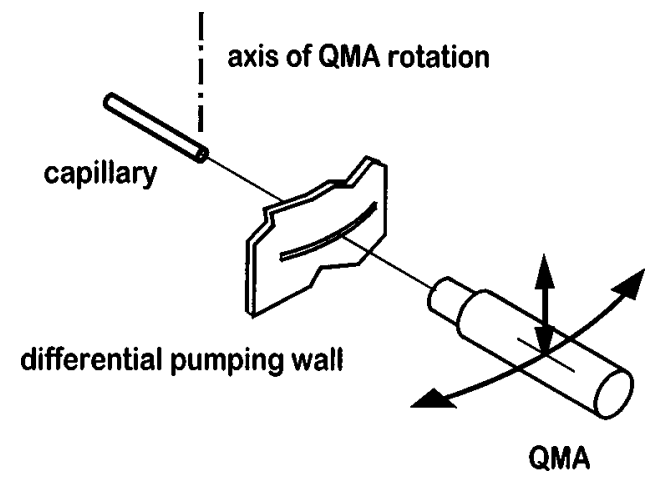

FIG. 2. Schematic arrangement of the quadrupole mass analyzer (QMA). It is translated vertically to measure the cracking factor and rotated horizontally to measure the angular distribution.

sured temperature is estimated to be valid along about 30 $\mathrm{mm}$ of the capillary up to its orifice ${ }^{22}$ and is termed the nominal temperature of the capillary.

In addition to the capillary temperature, the flow rate of hydrogen gas to the source was intended to be a parameter of the measurements. The lower flow rates applied in this experiment are too small to be handled by a mass flow controller. We therefore use a leak valve for flow control and adjust and measure the pressure in the gas feed line to the source. The higher feeding pressures are measured with a capacitance manometer and the lower ones with a spinning rotor gauge. The flow rate can be evaluated as the product of the feeding pressure and the flow conductance of the capillary. The latter is determined experimentally, which will be described in more detail below.

The degree of dissociation and the angular distribution of the hydrogen atoms are evaluated from the ion currents of a QMA as recorded with a Faraday cup at mass units 1 and 2. The QMA (Balzers, QMA 125) comprises an axial beam ion source, a 100-mm-rod system, and the Faraday cup. It is suspended from a combined linear and rotary feedthrough and can be translated and rotated as sketched in Fig. 2. The QMA is differentially pumped to reduce the background pressure. Nevertheless, the residual molecular hydrogen in the QMA chamber and also the molecular hydrogen effusing at lower temperatures from the capillary noticeably contribute to the signal at mass unit 1 by cracking. The cracking factor is determined by shifting the QMA into the shadow of the differential pumping wall where the signal at mass unit 1 solely originates from the cracking of molecular hydrogen in the ionizer. The respective $\mathrm{H}_{1}$ to $\mathrm{H}_{2}$ signal ratio is the cracking factor. When the QMA is exposed to the beam the $\mathrm{H}_{2}$ signal is multiplied by the cracking factor to obtain the background, which has to be subtracted from the measured $\mathrm{H}_{1}$ signal to obtain the proper atomic beam $\mathrm{H}_{1}$ signal.

The degree of dissociation is derived from the dependence of the beam $\mathrm{H}_{1}$ signal on the capillary temperature. The angular distribution is measured by rotating the QMA around the capillary orifice up to $15^{\circ}$. The angular distribution data and their analytical treatment were dealt with in our previous article. ${ }^{22}$

The angular resolution is limited by the ionizer of the QMA. From the variation of the beam signal in the shadow 


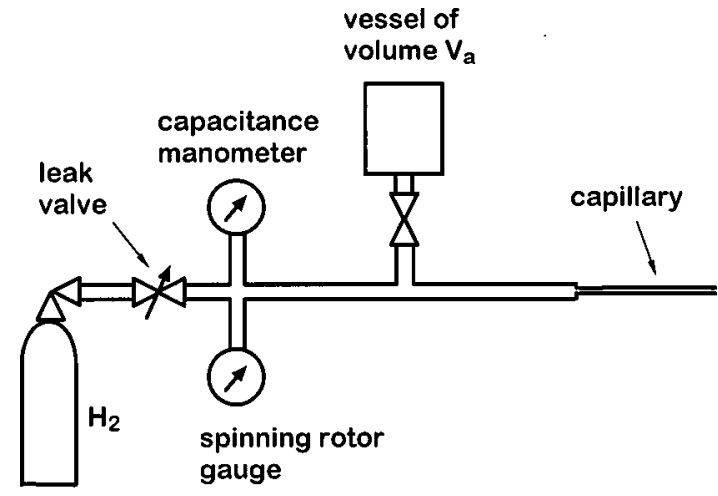

FIG. 3. Schematic drawing of the gas feed to the capillary.

of a nonpermanent aperture (not shown in Fig. 2) we estimate the cross section of the effective ionizing volume to be a circle $2.4 \mathrm{~mm}$ in diameter. This volume is located around $70 \mathrm{~mm}$ away from the capillary orifice as we judge from the geometry of the ionizer and the applied potentials. (This measure is felt to be more useful than the location of the ionizer center noted in our previous article. ${ }^{22}$ ) The diameter of the effective cross section corresponds to a plane angle of $2^{\circ}$ as seen from the orifice and the respective area to a solid angle of around $1 \times 10^{-3}$ sr. For comparison, the gold foil of Eibl et al. ${ }^{20}$ covers around $8^{\circ}$ and $20 \times 10^{-3} \mathrm{sr}$, respectively.

\section{EXPERIMENTAL RESULTS AND FORMALISM FOR THE EVALUATION OF THE INTENSITY}

\section{A. Gas flow rate}

The flow rate $\dot{Q}$ of hydrogen gas to the source can be expressed in terms of the feeding pressure $p$ and the flow conductance $C$ of the capillary. The conductance of the gas feed line between the leak valve and the capillary is much larger than that of the capillary. The pressure in the gas line is accordingly assumed to be uniform. If the pressure of the chamber into which the gas emanates is low as compared with the feeding pressure, the flow rate is given by

$$
\dot{Q}=C p \text {. }
$$

The feeding pressure $p$ is measured, whereas the flow conductance $C$ has to be determined separately. This can be performed by a transient flow experiment, i.e., by draining a vessel of known volume through the capillary and measuring the rate of the pressure change in the vessel. Closing the leak valve establishes a vessel of a defined volume $V_{m}$ comprising the manifold between the leak valve and the capillary. This volume, however, cannot be easily determined due to its complexity. Therefore, an additional vessel of known volume $V_{a}$ is attached to the gas feed manifold via a valve as shown schematically in Fig. 3. With the additional vessel valved off or on we open the leak valve until a stationary flow is obtained, whereupon we close the leak valve and monitor the decreasing pressures $p_{m}(t)$ and $p_{m a}(t)$, respectively, as a function of time. The rates of change of the momentary amounts of gas in the volumes $V_{m}$ and $V_{m}+V_{a}$ are, respectively,

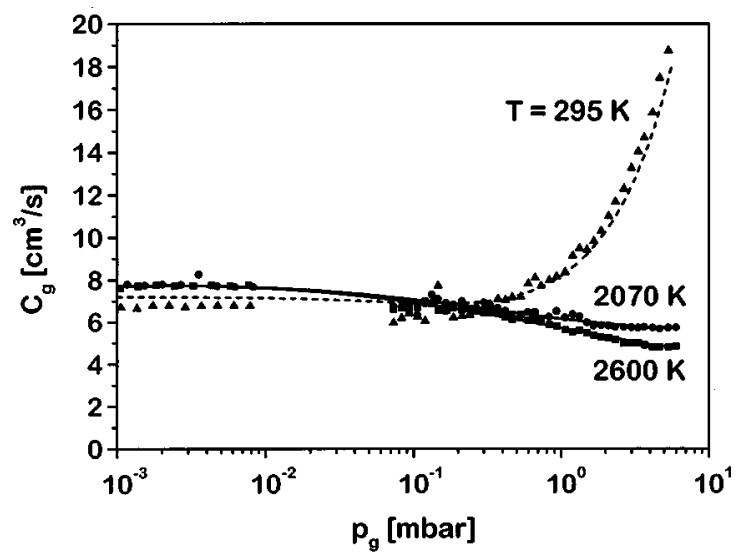

FIG. 4. The flow conductance of the capillary as measured at room temperature and at two elevated temperatures. The dotted line is the conductance as calculated by the Knudsen formula, Eq. (4), for the capillary at room temperature.

$$
\begin{aligned}
& V_{m} \dot{p}_{m}=-C p_{m}, \\
& \left(V_{m}+V_{a}\right) \dot{p}_{m a}=-C p_{m a} .
\end{aligned}
$$

Since, in general, the conductance is a function of the pressure, $C=C(p)$, we consider $\dot{p}_{m}(p)$ and $\dot{p}_{m a}(p)$ as functions of the pressure $p_{m}=p_{m a}=p$. By eliminating $V_{m}$ from Eqs. (2a) and (2b) we obtain the conductance of the capillary (note that $V_{m}<V_{m}+V_{a}$ and therefore $\dot{p}_{m}<\dot{p}_{m a}<0$ )

$$
C(p)=\frac{V_{a}}{p}\left(\frac{1}{\dot{p}_{m}(p)}-\frac{1}{\dot{p}_{m a}(p)}\right)^{-1} .
$$

The conductance measured as described above is termed $C_{g}$ for reasons given below and is shown in Fig. 4 for different capillary temperatures, i.e., for room temperature, for a lower operating temperature of $2070 \mathrm{~K}$, and for the highest temperature of $2600 \mathrm{~K}$. Pressures below $0.001 \mathrm{mbar}$ are measured by the spinning rotor gauge and the higher pressures by the capacitance manometer. There is a gap in the experimental data between 0.001 and 0.004 mbar and an increased scatter of the capacitance manometer data at the low end of their range where, however, the variation of conductance with pressure is small. At low pressures the conductance is independent of the pressure, which is indicative of molecular flow in the capillary, and the variation with temperature is small. At high pressures the low and high temperature branches behave qualitatively differently. The room temperature branch increases steeply at pressures above 1 mbar signifying viscous flow, whereas the high temperature branches decline with increasing pressure.

To explain at least qualitatively the dependence of the conductance on pressure and temperature we refer to the Knudsen formula, ${ }^{25,26}$ which describes the conductance in the pressure range considered here by

$$
C=\frac{\pi}{8} \frac{R^{4}}{L} \frac{\bar{p}}{\eta}+Z \frac{2 \pi}{3} \frac{R^{3}}{L} \bar{c},
$$

with

$$
Z=\frac{1+2 y}{1+2.47 y}
$$


and

$$
y=\sqrt{\frac{8}{\pi}} R \frac{\bar{p}}{\bar{c} \eta},
$$

where $R=0.5 \mathrm{~mm}$ is the inner radius of the capillary, $L$ $=64 \mathrm{~mm}$ is its length, $\eta$ is the viscosity of the gas, $\bar{p}$ the mean pressure in the capillary, and $\bar{c}$ the mean thermal speed of the gas molecules.

The conductance at room temperature, $T_{R}=295 \mathrm{~K}$, where the viscosity is $\eta=88.7 \times 10^{-6} \mathrm{~g} / \mathrm{cm} \mathrm{s}$ (Ref. 27), is calculated by Eq. (4) and shown by the dotted line in Fig. 4. The calculated conductance matches the experimental data rather well. If the experimental data were used to determine an effective inner radius of the capillary from Eq. (4), the lower pressure branch would give a radius $2 \%$ smaller and the higher pressure branch a radius $2 \%$ larger than the nominal radius of $0.5 \mathrm{~mm}$.

The Knudsen formula cannot be strictly applied to the hot capillary because the temperature is not homogeneous but varies from near room temperature at the water-cooled socket to temperatures above $2000 \mathrm{~K}$ near the orifice. Furthermore, the particle flow is not constant along the capillary if dissociation occurs. To make at least a qualitative use of the Knudsen formula the hot capillary may be approached by a model capillary characterized by a homogeneous temperature and the absence of dissociation. Varying the temperature of this model capillary from room temperature to higher temperatures and taking the pressure jump at its entrance due to thermal transpiration ${ }^{28}$ into account, we find the conductance to be invariant at low pressures and to decrease at high pressures. The latter is due to the increase of the viscosity with temperature. These results explain the experimental data qualitatively but not quantitatively. A rigorous calculation has to include the temperature variation along the capillary as well as dissociation. This, however, is outside the scope of the present article.

From the experimental data of the capillary conductance and the feeding pressure the gas flow rate can now be determined by Eq. (1). To evaluate the flow of molecules $\dot{N}$ from this one has to pay attention to the gas temperature. It is advantageous that both our gauges are "cold" and do not influence the gas temperature, which is accordingly equal to the room temperature $T_{R}$. To be specific we henceforth write $p_{g}, \dot{Q}_{g}, C_{g}$, and $\dot{N}_{g}$, where the index $g$ refers to the gauges. When the source is running continuously and the flow is stationary, the flow rate is $\dot{Q}_{g}=\dot{V}_{g} p_{g}$, where $\dot{V}_{g}=C_{g}$ is the volumetric flow past the gauges [cf. Eq. (1)]. Finally, we obtain the number of hydrogen molecules passing the gauges at unit time $\dot{N}_{g}$ from the relation $\dot{N}_{g}=n_{g} \dot{V}_{g}$ and the ideal gas law $p_{g}=n_{g} k T_{R}$,

$$
\dot{N}_{g}=\frac{p_{g} C_{g}}{k T_{R}},
$$

where $n_{g}$ is the number density of the gas at the position of the gauges and $k$ is the Boltzmann constant.

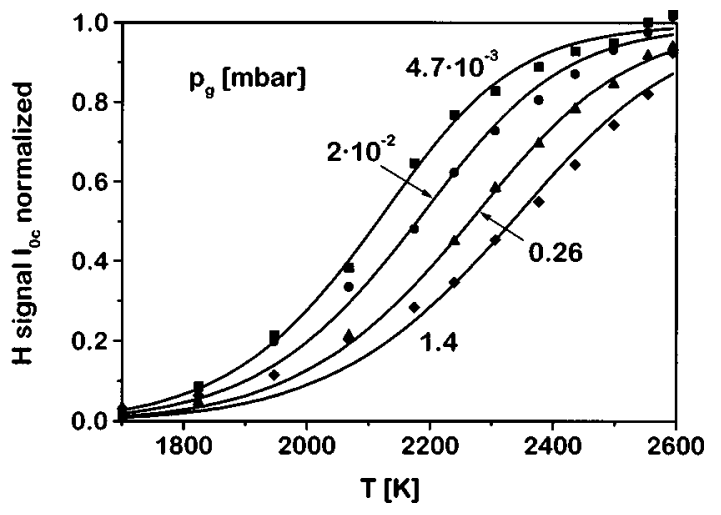

FIG. 5. The signal of atomic hydrogen as measured on the axis of the capillary at various capillary temperatures. The signal is corrected for the varying velocity of the atoms and normalized. The solid lines are calculated by an equilibrium model [Eqs. (12), (7), and (8)].

\section{B. Degree of dissociation}

The higher the temperature of the capillary the greater should be the portion of the hydrogen gas which dissociates. A quantitative description of this dependence will be given in this section. To monitor the degree of dissociation the feeding pressure $p_{g}$ remains constant and the QMA ion currents at mass units 1 and 2 are measured on the capillary axis as well as in the wall shadow for varying temperatures. Subtracting the background from the on-axis $\mathrm{H}_{1}$ signal as described in Sec. II we obtain the on-axis beam signal $I_{0}$.

Beam signals measured at different capillary temperatures have to be corrected for the velocity of the hydrogen atoms, which determines the time the atoms spend in the ion source of the QMA and consequently the probability of their ionization. $^{29}$ This is accounted for by multiplying the beam signal $I_{0}$ by the factor $\sqrt{T / T_{R}}$, which is the ratio of the mean velocities at $T$ and $T_{R}$. The velocity-corrected beam signal is denoted $I_{0 c}$. It is proportional to the center-line intensity of the atomic hydrogen beam.

Figure 5 shows the signals $I_{0 c}$ for different feeding pressures. The signals tend to saturate at high temperatures and are accordingly normalized to the saturation value. The fitting solid lines will be treated below. The data show a rather sensitive dependence on the capillary temperature and a weak dependence on the pressure, which is similar to the dependence of the degree of equilibrium dissociation on gas temperature and pressure.

However, the data cannot be directly identified with the degree of equilibrium dissociation in the source. To apply the theory of equilibrium dissociation we will now consider a simplified model of the capillary intended to represent the capillary with respect to the dissociation processes involved. Not far from the orifice, we insert into the capillary a virtual chamber which is at the nominal temperature of the capillary and where the gas is in thermal equilibrium with the chamber walls. The part of the capillary between this chamber and the orifice is assumed not to contribute to dissociation and molecular flow is presumed in this part of the capillary.

The degree of equilibrium dissociation $\alpha$ in the virtual chamber is defined by the particle densities of hydrogen atoms and molecules $n_{1}$ and $n_{2}$, respectively, via 


$$
\alpha \equiv \frac{n_{1}}{n_{1}+2 n_{2}}
$$

It is a function of the temperature $T$ and pressure $p_{\text {equ }}$ in the virtual chamber according to

$$
\alpha=\sqrt{\frac{K_{p}}{4 \frac{p_{\text {equ }}}{p^{0}}+K_{p}}},
$$

where $p_{\text {equ }}=p_{1}+p_{2}$ is the total pressure and $p_{1}$ and $p_{2}$ are the partial pressures of atoms and molecules, respectively. ${ }^{30}$ The equilibrium constant $K_{p}$ is defined by $K_{p} \equiv p_{1}^{2} /\left(p_{2} \cdot p^{0}\right)$ $\left(p^{0}=1 \mathrm{bar}\right),{ }^{31}$ and depends solely on the temperature. It is tabulated $^{32}$ and can, in the temperature range of interest, be calculated from the fit function

$$
\log K_{p}=6.304-\frac{23760 \mathrm{~K}}{T} .
$$

The fluxes of atoms and molecules leaving the capillary, $\dot{N}_{1}$ and $\dot{N}_{2}$, respectively, are related to the molecule flux $\dot{N}_{g}$ passing the gauges by the mass conservation law

$$
2 \dot{N}_{g}=\dot{N}_{1}+2 \dot{N}_{2} \text {. }
$$

To relate the fluxes $\dot{N}_{1}$ and $\dot{N}_{2}$ out of the capillary to the densities $n_{1}$ and $n_{2}$ in the virtual chamber we consider the molecular flow from the virtual chamber through the downstream capillary section. The particle fluxes passing this capillary section are

$$
\dot{N}_{i}=\left(\frac{1}{4} n_{i} \bar{c}_{i}\right) \cdot F W, \quad(i=1,2),
$$

where $\bar{c}_{i}$ is the mean thermal velocity in the virtual chamber and the term in parentheses is the thermal flux density onto the entrance of the capillary section whose cross section is $F$. $W$ is the probability of a gas particle passing the section and leaving the capillary. $W$ is independent of the type of gas if the scattering of the gas particles at the capillary wall is free of a specular component as is commonly assumed in the theory of molecular flow. ${ }^{33}$

Using $n_{2}$ as derived from Eq. (6) and inserting Eq. (10) for $i=2$ into Eq. (9) we obtain

$$
2 \dot{N}_{g}=\dot{N}_{1}+\frac{1}{2} \frac{1-\alpha}{2 \alpha} n_{1} \bar{c}_{2} F W=\dot{N}_{1}+\frac{1-\alpha}{\alpha} \frac{\dot{c}_{2}}{\dot{c}_{1}} \dot{N}_{1},
$$

which yields

$$
\dot{N}_{1}=2 \dot{N}_{g} \frac{\sqrt{2} \alpha}{1-\alpha+\sqrt{2} \alpha} \text {. }
$$

where we made use of $\bar{c}_{1} / \bar{c}_{2}=\sqrt{2}$. Equation (11) relates the total flux of atomic hydrogen emitted from the source to the flux of hydrogen molecules entering the source and the degree of dissociation in the virtual chamber.

The temperature dependence of the velocity-corrected beam signal $I_{0 c}$ is derived from Eqs. (11) and (5) as follows. As stated above, $I_{0 c}$ is proportional to the center-line intensity of the atomic hydrogen beam. This intensity is proportional to the total flux $\dot{N}_{1}$ provided that the angular distribution does not vary in the temperature range of interest, i.e., from 1800 to $2600 \mathrm{~K}$. We have no experimental data on this matter since we measured the angular distribution at $2600 \mathrm{~K}$ only. ${ }^{22}$ For simplicity we assume the angular distribution to be independent of the temperature. In this approximation, $I_{0 c}$ is proportional to the total flux $\dot{N}_{1}$ and has the temperature dependence included in Eq. (11). The factor $\dot{N}_{g}$ in Eq. (11) was determined in the former section and found to be dependent on $p_{g}$ and $C_{g}$ [Eq. (5)]. Since $p_{g}$ remains constant and $C_{g}$ hardly varies with temperature (Fig. 4) $\dot{N}_{g}$ can be assumed to be constant for every data set of Fig. 5. Consequently, $I_{0 c}$ is proportional to the fractional term in Eq. (11), which increases from 0 to 1 when $\alpha$ is varied from 0 to 1 . Since $I_{0 c}$ as plotted in Fig. 5 is also normalized to unity we have explicitly

$$
I_{0 c, \text { normalized }}=\frac{\sqrt{2} \alpha}{1-\alpha+\sqrt{2} \alpha} .
$$

The solid lines in Fig. 5 are calculated from this equation and from the expression for $\alpha$ given by Eqs. (7) and (8). In Eq. (7) a proper $p_{\text {equ }}$ was chosen for every feeding pressure $p_{g}$ to fit the experimental data. More precisely, two parameters were varied in the same fitting process, i.e., the saturation QMA signal to which the signal $I_{0 c}$ has been normalized and the pressure $p_{\text {equ }}$. Varying the first parameter stretches the data along the $y$ axis, varying the second shifts the fitting lines along the $x$ axis.

With the present model of equilibrium dissociation, a satisfying description of the experimental data has been obtained. For a constant feeding pressure the variation of the dissociation efficiency with temperature is described by a single parameter. Values of this parameter $p_{\text {equ }}$ are given in Table I. Knowing $p_{\text {equ }}$ we can calculate $\alpha$ from Eqs. (7) and (8) and obtain the total flux $N_{1}$ of atomic hydrogen by Eq. (11).

\section{Angular distribution}

The third quantity needed to evaluate the intensity of the source is the angular distribution of the hydrogen atoms. Denoting the polar angle with respect to the capillary axis by $\vartheta$ and the differential solid angle with respect to the orifice center by $d \omega$, the angularly resolved distribution of atomic hydrogen $J(\vartheta)$ in units of atoms/sr s can be written as the product of the center-line intensity $J_{0}$ given in atoms/sr s and the dimensionless normalized angular distribution $j(\vartheta)$

$$
J(\vartheta) d \omega=J_{0} j(\vartheta) d \omega .
$$

Note that $j(0)=1$. In our previous article ${ }^{22}$ we reported data on the normalized angular distribution of the source measured for the same feeding pressures as applied in the present dissociation measurements. The experimental distributions were described by an analytic expression $j(\vartheta)$. Combining Eqs. (2), ${ }^{34}(3),(4),(6)$, and (7) from that work we obtain 
TABLE I. Parameters characterizing the source of atomic hydrogen when operated at $2600 \mathrm{~K}$.

\begin{tabular}{|c|c|c|c|c|c|c|c|c|c|c|}
\hline $\begin{array}{l}\text { Feeding } \\
\text { pressure } \\
p_{g} \\
\text { (mbar) }\end{array}$ & $\begin{array}{c}\text { Conductance } \\
C_{g} \\
\left(\mathrm{~cm}^{3} / \mathrm{s}\right)\end{array}$ & $\begin{array}{c}\text { Gas flow } \\
\text { rate } \dot{Q}_{g} \\
(\operatorname{mbar} \ell / \mathrm{s})\end{array}$ & $\begin{array}{c}\text { Mass flow } \\
\text { rate } \dot{N}_{g} \\
\text { (molecules/s) }\end{array}$ & $\begin{array}{l}\text { Virtual } \\
\text { equilibrium } \\
\text { pressure } p_{\text {equ }} \\
\text { (mbar) }\end{array}$ & $\begin{array}{c}\text { Degree of } \\
\text { dissociation } \\
\alpha\end{array}$ & $\begin{array}{c}\text { Total flux } \\
\text { of } \\
\text { H atoms } \\
\dot{N}_{1} \\
\text { (atoms/s) }\end{array}$ & $\begin{array}{l}\text { Effective } \\
\text { length } \\
\text { of } \\
\text { transparent } \\
\text { flow } \\
l_{\text {eff }}\end{array}$ & $\begin{array}{c}\text { Integral } \\
\text { of } \\
\text { angular } \\
\text { distribution } \Omega \\
(\mathrm{sr})\end{array}$ & $\begin{array}{c}\text { On-axis } \\
\text { intensity }^{\mathrm{a}} \\
\text { of } \\
\mathrm{H} \text { atoms } \\
J_{0} \\
\text { (atoms/s sr) }\end{array}$ & $\begin{array}{c}\text { Flux density } \\
\text { of } \mathrm{H} \text { atoms } \\
\text { on a } \\
\text { sample } \\
\text { at } d=8 \mathrm{~cm} \\
\varphi_{0} \\
\text { (atoms } / \mathrm{cm}^{2} \mathrm{~s} \text { ) }\end{array}$ \\
\hline 0.0047 & 7.7 & $3.62 \times 10^{-5}$ & $8.89 \times 10^{14}$ & 0.014 & 0.981 & $1.75 \times 10^{15}$ & 13 & 0.267 & $6.57 \times 10^{15}$ & $1.0 \times 10^{14}$ \\
\hline 0.02 & 7.5 & $1.50 \times 10^{-4}$ & $3.68 \times 10^{15}$ & 0.03 & 0.961 & $7.17 \times 10^{15}$ & 11.4 & 0.302 & $2.37 \times 10^{16}$ & $3.7 \times 10^{14}$ \\
\hline 0.26 & 6.5 & $1.69 \times 10^{-3}$ & $4.15 \times 10^{16}$ & 0.08 & 0.906 & $7.73 \times 10^{16}$ & 7 & 0.474 & $1.63 \times 10^{17}$ & $2.6 \times 10^{15}$ \\
\hline 1.4 & 5.5 & $7.70 \times 10^{-3}$ & $1.89 \times 10^{17}$ & 0.16 & 0.834 & $3.32 \times 10^{17}$ & 3.8 & 0.805 & $4.12 \times 10^{17}$ & $6.4 \times 10^{15}$ \\
\hline
\end{tabular}

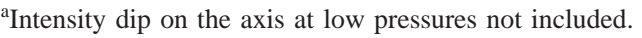

$$
\begin{aligned}
j(\vartheta)= & U(\beta) \cos \vartheta+\frac{4}{3 \pi}\left(1-\frac{1}{2 l_{\mathrm{eff}}+1}\right) \frac{1}{l_{\mathrm{eff}}}[1-V(\beta)] \\
& \times \frac{\cos ^{2} \vartheta}{\sin \vartheta}+\frac{1}{2 l_{\mathrm{eff}}+1}[1-U(\beta)] \cos \vartheta \\
= & \left\{U(\beta)+\frac{1}{2 l_{\mathrm{eff}}+1}\left[\frac{8}{3 \pi} \frac{1-V(\beta)}{\operatorname{tg} \vartheta}\right.\right. \\
& +1-U(\beta)]\} \cos \vartheta
\end{aligned}
$$

with

$$
\left\{\begin{array}{l}
U(\beta)=\frac{(2 \beta-\sin 2 \beta)}{\pi}, \quad V(\beta)=\sin ^{3} \beta, \quad \text { for } \vartheta<\operatorname{arctg} \frac{1}{l_{\mathrm{eff}}} \\
U(\beta)=V(\beta)=0, \quad \text { for } \quad \vartheta \geqslant \operatorname{arctg} \frac{1}{l_{\mathrm{eff}}},
\end{array}\right.
$$

where $\beta=\beta(\vartheta)$ is defined by $\cos \beta=l_{\text {eff }} \operatorname{tg} \vartheta$. The parameter $l_{\text {eff }}$ is the only fitting parameter. It depends on the feeding pressure. In our theoretical model of the beam formation in the capillary, $l_{\text {eff }}$ is the effective length of transparent flow divided by the capillary diameter.

We now benefit from the former derivation of an analytic expression for $j(\vartheta)$, which makes it feasible to extrapolate the measured angular distribution and to integrate $j(\vartheta)$ over the hemisphere into which hydrogen atoms are emitted. This integral is denoted by $\Omega$,

$$
\Omega \equiv \int_{2 \pi} j(\vartheta) d \omega .
$$

The unit of $\Omega$ is sr and the integral can be evaluated numerically or using a formula given by Zugenmaier. ${ }^{35}$ Since $\int_{2 \pi} J(\vartheta) d \omega=\dot{N}_{1}$ we obtain by integration of Eq. (13):

$$
J_{0}=\frac{\dot{N}_{1}}{\Omega} .
$$

Finally, we obtain from Eqs. (13) and (16) and by substituting $\dot{N}_{1}$ from Eqs. (11) and (5)

$$
J(\vartheta) d \omega=\frac{p_{g} C_{g}}{k T_{R}}\left(2 \frac{\sqrt{2} \alpha}{1-\alpha+\sqrt{2} \alpha}\right) \frac{j(\vartheta)}{\Omega} d \omega .
$$

Equation (17) is written as the product of three specific factors which reflect the present route to the determination of the intensity of the source. The first factor is the rate at which hydrogen molecules enter the source. It is here evaluated from the feeding pressure and the conductance of the capillary. The second factor describes the efficiency of dissociation in the source and includes an apparent degree of dissociation $\alpha$ which can be calculated from the capillary temperature and the experimental parameter $p_{\text {equ }}$. The maximum value of the second factor is 2 corresponding to two atoms per molecule. The third factor specifies the angular distribution of the atoms leaving the source. It can be calculated from an analytic expression involving the experimental parameter $l_{\text {eff }}$.

\section{EVALUATION OF THE INTENSITY AND THE PERFORMANCE OF THE SOURCE}

So far the raw data and the formalism for the evaluation of the intensity have been presented. In this section we will use the relations derived above to enumerate the characteristic parameters of the source, which are summarized in Table I. All the data in Table I depend on the feeding pressure $p_{g}$ and are arranged accordingly. Except for the experimental parameters $p_{\text {equ }}$ and $l_{\text {eff }}$, all the data depend furthermore on the temperature and are given for $T=2600 \mathrm{~K}$. We will discuss the data of Table I by column.

The flow conductance $C_{g}$ of the capillary is taken from Fig. 4. The gas flow $\dot{Q}_{g}$ is simply the product of $C_{g}$ and $p_{g}$, see Eq. (1). If the source is operated in a vacuum system with known pumping speed for hydrogen, the gas flow $\dot{Q}_{g}$ can also be evaluated as the product of the pumping speed and the pressure in the system provided there is no other source or sink of hydrogen. The number $\dot{N}_{g}$ of hydrogen molecules passing the gauges at unit time results from Eq. (5) with $T_{R}=295 \mathrm{~K}$. Like the gas flow $\dot{Q}_{g}$, this number is almost proportional to the feeding pressure $p_{g}$, since the conductance $C_{g}$ varies only moderately with $p_{g}$.

The fitting parameter $p_{\text {equ }}$ determines the degree of dissociation at a given temperature. Note that $p_{\text {equ }}$ is greater than $p_{g}$ in the first two rows of Table I. At first sight, this seems to be inconsistent with the assumption of a virtual chamber at pressure $p_{\text {equ }}$ somewhere along the capillary. However, at molecular flow the pressure in the capillary de- 


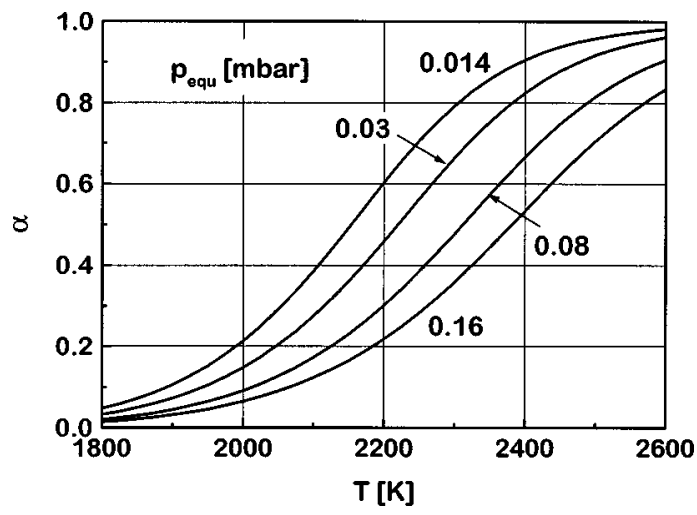

FIG. 6. The degree of dissociation $\alpha$ as calculated by Eqs. (7) and (8) for the equilibrium pressures $p_{\text {equ }}$ indicated in the figure. The lines are similar to those of Fig. 5 but not identical.

pends strongly on the temperature due to thermal transpiration. ${ }^{28}$ Along a certain part of the capillary adjacent to the socket where the temperature rises steeply with distance from the socket, the pressure can be significantly higher than $p_{g}$.

The degree of dissociation $\alpha$ can be calculated from $p_{\text {equ }}$ by Eqs. (7) and (8) and is plotted in Fig. 6. The curves are similar in shape to, but not identical with, those of Fig. 5. Values of $\alpha$ for $T=2600 \mathrm{~K}$ are given in Table I. At this temperature the degree of dissociation is close to $100 \%$ at the lowest feeding pressure and still higher than $80 \%$ at the highest pressure. The number of hydrogen atoms $\dot{N}_{1}$ leaving the capillary per unit time can now be calculated by Eq. (11). These numbers are almost twice the number $\dot{N}_{g}$ of passing molecules since the degree of dissociation is high.

The fitting parameter $l_{\text {eff }}$ determines the normalized angular distribution which is described by Eq. (14) and is narrower the greater $l_{\text {eff }}$ is. ${ }^{22}$ Numerical values of $l_{\text {eff }}$ were reported in our previous article. ${ }^{22}$ However, further measurements after improving the differential pumping of the QMA revealed that the intensity dip on the axis, which we clearly resolved only for the lowest feeding pressure, ${ }^{22}$ also occurs at higher pressures although it is less pronounced. We therefore reconsidered the fit of the experimental data for the two lowest pressures and obtained the somewhat larger values of $l_{\text {eff }}$ listed in Table I.

A comment on the dip is appropriate here. It was first reported by Van Zyl and Gealy ${ }^{18}$ and its origin was clearly illustrated by the Monte Carlo simulations of Eibl et al. ${ }^{20}$ The dip is due to the limited length of the hot section of the capillary. Since the hot section in Bischler and Bertel's source is shorter than in ours, the reduced lengths (length divided by diameter) being around 8 and 30, respectively, the intensity dip of that source should accordingly be more pronounced. The fact that Eibl et $a .^{20}$ did not observe a dip in their measurements with Bischler and Bertel's source can only be attributed to their moderate angular resolution mentioned above.

Since the inner diameter of the capillary is $1 \mathrm{~mm}, l_{\text {eff }}$ can also be read as the effective length of transparent flow in units of $\mathrm{mm}$. It is plausible that the transparent flow is more extended the lower the feeding pressure $p_{g}$ is. A quantitative

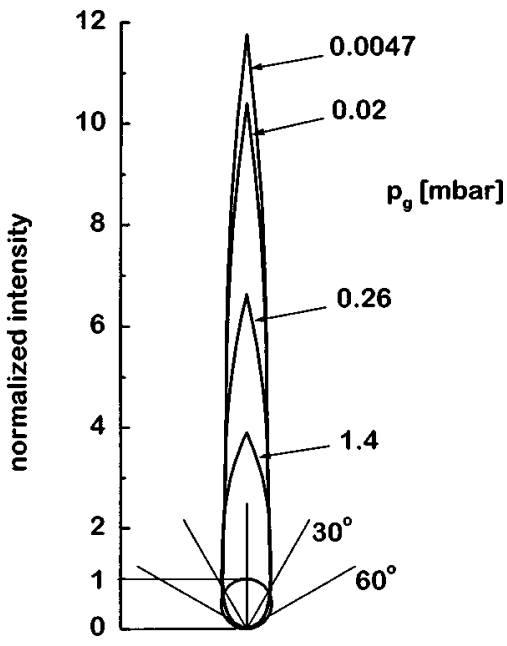

FIG. 7. Polar plot of the angular distribution of atomic hydrogen emitted from the capillary at a temperature of $2600 \mathrm{~K}$ at different feeding pressures $p_{g}$. The distributions are scaled in such a way as to superimpose the cosine distributions of thin-walled apertures passed by the same total flux. This normalization makes all the cosine distributions coincide with the circle at the bottom. The intensity dip on the axis at low pressures is not included. The figure elucidates the beam formation by the capillary.

investigation of this dependence, however, would require a calculation of the pressure profile along the capillary, which is hampered by the variation of the temperature and by the event of dissociation.

With respect to Eq. (16), $\Omega$ can be thought of as the solid angle which would contain the total flux if the intensity were isotropic in that solid angle and equal to the center-line intensity $J_{0} . \Omega$ is obviously a measure of the width of the angular distribution. By means of $\Omega$ we can evaluate the enhancement $F$ of the center-line intensity of the source as contrasted with that of a thin-walled aperture. For the aperture we have $J_{\text {aperture }}(\vartheta)=J_{0 \text {,aperture }} \cdot \cos (\vartheta)$ and $\Omega_{\text {aperture }}$ $=\pi$ sr. Assuming the same total flux $\dot{N}_{1}$ is emitted from both the capillary and the aperture we obtain from Eq. (16)

$$
F=\frac{J_{0, \text { capilary }}}{J_{0, \text { aperture }}}=\frac{\pi \mathrm{sr}}{\Omega} .
$$

Figure 7 illustrates the enhancement and shows the angular intensity distribution in a polar plot. The distributions for the different feed pressures are calculated by Eqs. (14) and (18) with $\Omega$ and $l_{\text {eff }}$ as given in Table I. According to Eq. (18) each distribution is normalized to the on-axis intensity of a thin-walled aperture passed by the same total flux. This normalization makes the distribution of the aperture a circle of unit diameter irrespective of the total flux. The distributions of the capillary are markedly elongated. The height as read on the vertical scale is the enhancement factor $F$. These distributions illustrate the beam formation by molecular flow in the capillary, which is treated in our previous article. ${ }^{22}$

The on-axis intensity $J_{0}$ as evaluated by Eq. (16) is plotted in Fig. 8(a). If the intensity were proportional to the pressure $p_{g}$ it would run along the dotted line. In order to visualize the reason for the slower rise we combine Eqs. (16) and (11) to obtain 

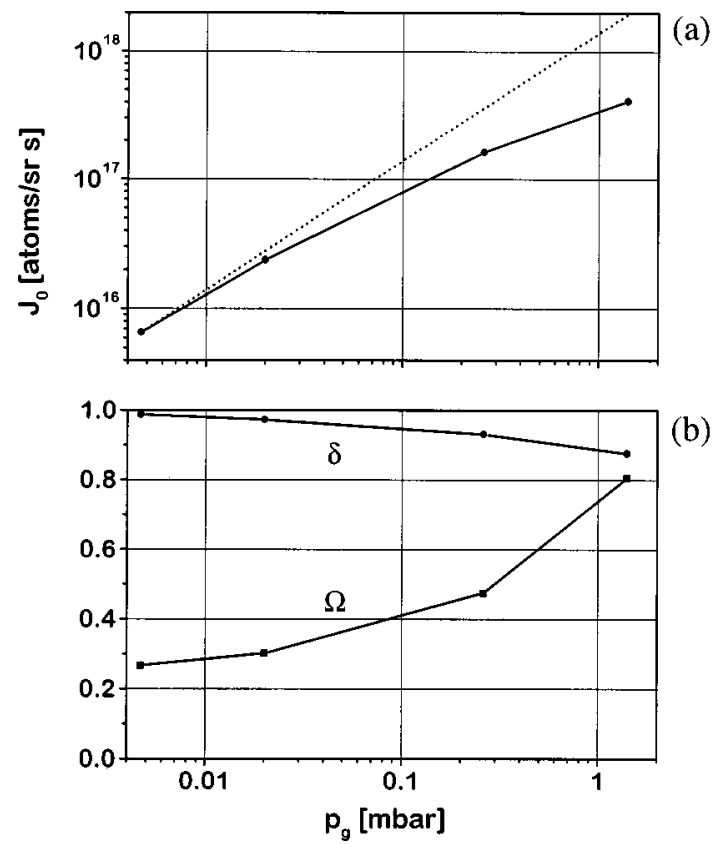

(b)

FIG. 8. (a) The on-axis flux density $J_{0}$ of atomic hydrogen as a function of the feeding pressure $p_{g}$. The dotted line represents a linear increase of $J_{0}$ with $p_{g}$. The deviation of the experimental data from linearity originates from the variation of the flow conductance and the two factors plotted in (b), where $\delta$ is a measure of the degree of dissociation and $\Omega$ a measure of the angular distribution, cf. Eq. (19).

$$
J_{0}=2 \dot{N}_{g} \frac{\delta}{\Omega},
$$

with

$$
\delta=\frac{\sqrt{2} \alpha}{1-\alpha+\sqrt{2} \alpha} .
$$

The factor $\dot{N}_{g}$ is almost proportional to $p_{g}$ as mentioned above. The quantities $\delta$ and $\Omega$ reflect the degree of dissociation and the width of the angular distribution, respectively, and are plotted in Fig. 8(b). They both contribute to the slower rise of the intensity at higher feeding pressures but the variation of $\Omega$ is much stronger than that of $\delta$. Accordingly, the deterioration of the intensity is mainly due to the less efficient beam formation at higher pressures.

The final and for the user most illustrative feature of the source is the flux density $\varphi$ on a sample to be exposed to atomic hydrogen. The flux density $\varphi_{0}$ on a sample positioned on the capillary axis at distance $d$ from the end of the capillary is simply

$$
\varphi_{0}=\frac{J_{0} \mathrm{sr}}{d^{2}} .
$$

The flux density $\varphi_{0}$ is proportional to $J_{0}$ and values for $d$ $=8 \mathrm{~cm}$ are listed in Table I. The flux densities $\varphi_{0}$ range up to well above a monolayer per second. The data in Table I do not consider the intensity dip on the axis at low feeding pressures. Taking this dip into account $\varphi_{0}$ is reduced by factor of 0.7 and 0.84 at $p_{g}=0.0047$ and 0.02 mbar, respectively.
Knowing the flux density, we can then evaluate the sensitivity of the QMA. Although this sensitivity is specific to the present apparatus its evaluation can serve as a check on the internal consistency of the data. We collected data on the angular distribution at $2600 \mathrm{~K}$ and data on the degree of dissociation measured at varying temperatures. How can the sensitivity of the QMA be consistently defined? In the case of the former data the sensitivity is appropriately defined as the ratio of the beam signal $I_{0}$ at $\vartheta=0$ and the center-line intensity $J_{0}$, where $I_{0}$ is measured in A and $J_{0}$ in atoms/sr s. This ratio is the sensitivity of the QMA for hydrogen atoms of a velocity corresponding to $2600 \mathrm{~K}$. We denote this sensitivity by $S_{2600}$. Reducing the velocity without altering the intensity would increase the QMA signal due to the longer time the atoms spend in the ion source. This is accounted for by the relation

$$
I_{0}(T)=S_{2600} \sqrt{\frac{2600 \mathrm{~K}}{T}} J_{0}(T),
$$

where $I_{0}$ and $J_{0}$ may now be determined at any temperature $T$. The dissociation data for a given pressure $p_{g}$ are accordingly evaluated by calculating $J_{0}(T)$ from the data in Table I and fitting the experimental data $I_{0}(T)$ by Eq. (21) using $S_{2600}$ as the fitting parameter. [Note that $I_{0}(T)$ represents the signal of the beam which is not yet velocity corrected, cf. Sec. III B] From each of the four different angular distributions and the four sets of dissociation data we obtain a value of the sensitivity $S_{2600}$. The mean of these eight numbers is $\bar{S}_{2600}=(2.08 \pm 0.06) \times 10^{-28} \mathrm{~A} / \mathrm{atoms} / \mathrm{sr}$ s. The standard error of the mean is only $3 \%$, which gives some confidence in the accuracy of the data and their evaluation.

\section{SUMMARY}

A source of atomic hydrogen based on a hot capillary is investigated. Quantification of the intensity is achieved by measuring the flow rate of the hydrogen feed gas in absolute numbers and by measuring the degree of dissociation and the angular distribution of the hydrogen atoms in relative numbers. The flow rate is determined via the measurement of the conductance of the capillary, which is evaluated from a pressure measurement and which at operating temperature is significantly different from that at room temperature. The degree of dissociation is derived from the signal of a quadrupole mass analyzer tuned to mass 1 and 2 and depends strongly on the temperature of the capillary. The experimental result is modeled assuming equilibrium dissociation and adopting a virtual equilibrium pressure. The measurement and analytic description of the normalized angular distribution was treated in a former article. The analytic description is used here to determine the integral of the normalized angular distribution, which enables the total flux and finally the angularly resolved intensity distribution to be evaluated. Due to beam formation in the capillary the center-line intensity is higher than that of a thin-walled aperture passed by the same total flux. The enhancement is about 12 at the lowest gas feed pressure of $4.7 \times 10^{-3}$ mbar and still about 4 at a pres- 
sure of 1.4 mbar. At this feeding pressure the flux density on a sample positioned $8 \mathrm{~cm}$ away from the capillary exit is about $6 \times 10^{15}$ atoms $/ \mathrm{cm}^{2} \mathrm{~s}$.

This flux density is determined at a capillary temperature of $2600 \mathrm{~K}$, which might be too high if the evaporation of tungsten has to be strictly avoided. We are presently testing a source, which is intended for somewhat lower temperatures and incorporates resistive heating instead of electron impact heating. This modification simplifies the design and operation of the source.

\section{ACKNOWLEDGMENTS}

The author is indebted to H. P. Fleischhauer for his skillful engineering and technical support. The helpful discussions with U. Littmark are gratefully acknowledged as well as the comments on the manuscript by him, T. SchwarzSelinger, H. Ibach, and J. Carter-Sigglow.

1 "Hydrogen in Semiconductors," edited by J. I. Pankove and N. M. Johnson, Semiconductors and Semimetals, Vol. 34, edited by R. K. Willardson and A. C. Beer (Academic, Boston, MA, 1991).

${ }^{2}$ Hydrogen in Semiconductors: Bulk and Surface properties, edited by M. Stutzmann and J. Chevalier, Physica B 170, (1991).

${ }^{3}$ Hydrogen in Compound Semiconductors, edited by S. J. Pearton, Mater. Sci. Forum 148/149 (1994)

${ }^{4}$ A. Sutoh, Y. Okada, S. Ohta, and M. Kawabe, Jpn. J. Appl. Phys., Part 2 34, L 1379 (1995)

${ }^{5}$ T. Sugaya and M. Kawabe, Jpn. J. Appl. Phys., Part 2 30, L 402 (1991). ${ }^{6}$ EPI MBE Products Group, 4900 Constellation Drive, St. Paul, MN 55127.

${ }^{7}$ W. L. Gardner, J. Vac. Sci. Technol. A 13, 763 (1995).

${ }^{8}$ E. C. Samano, W. E. Carr, M. Seidl, and B. S. Lee, Rev. Sci. Instrum. 64, 2746 (1993).

${ }^{9}$ M. Horn-von Hoegen and A. Golla, Surf. Sci. 337, L777 (1995).

${ }^{10}$ J. A. B. Hodgson and A. A. Haasz, Rev. Sci. Instrum. 62, 96 (1991).

${ }^{11}$ A. Donnelly, M. P. Hughes, J. Geddes, and H. B. Gilbody, Meas. Sci. Technol. 3, 528 (1992).
${ }^{12}$ R. W. McCullough, J. Geddes, A. Donnelly, M. Liehr, M. P. Hughes, and H. B. Gilbody, Meas. Sci. Technol. 4, 79 (1993).

${ }^{13}$ D. P. Higgins, R. W. McCullough, J. Geddes, J. M. Woolsey, M. Schlapp, and H. B. Gilbody, Key Eng. Mater. 99-100, 177 (1995).

${ }^{14}$ G. W. Wicks, E. R. Rueckwald, and M. W. Koch, J. Vac. Sci. Technol. B 14, 2184 (1996)

${ }^{15}$ W. L. Fite, R. Th. Brackmann, and W. R. Snow, Phys. Rev. 112, 1161 (1958).

${ }^{16}$ D. Spence and O. J. Steingraber, Rev. Sci. Instrum. 59, 2464 (1988).

${ }^{17}$ B. P. Paolini and M. A. Khakoo, Rev. Sci. Instrum. 69, 3132 (1998).

${ }^{18}$ B. Van Zyl and M. W. Gealy, Rev. Sci. Instrum. 57, 359 (1986).

${ }^{19}$ U. Bischler and E. Bertel, J. Vac. Sci. Technol. A 11, 458 (1993).

${ }^{20}$ C. Eibl, G. Lackner, and A. Winkler, J. Vac. Sci. Technol. A 16, 2979 (1998)

${ }^{21}$ I. Langmuir, J. Am. Chem. Soc. 34, 1310 (1912).

${ }^{22}$ K. G. Tschersich and V. von Bonin, J. Appl. Phys. 84, 4065 (1998).

${ }^{23}$ R. J. Thorn and G. H. Winslow, in Temperature-Its Measurement and Control in Science and Industry, edited by C. M. Herzfeld (Reinhold, New York, 1962), Vol. III, Part 1, Chap. 46.

${ }^{24}$ J. C. DeVos, Physica (Amsterdam) 20, 690 (1954).

${ }^{25}$ S. Dushman, Scientific Foundations of Vacuum Technique (Wiley, New York, 1962), p. 106.

${ }^{26}$ L. B. Loeb, Kinetic Theory of Gases (McGraw-Hill, New York, 1927), p. 262.

${ }^{27}$ M. J. Assael, S. Mixafendi, and W. A. Wakeham, J. Phys. Chem. Ref. Data 15, 1315 (1986).

${ }^{28}$ S. Dushman, Scientific Foundation of Vacuum Technique (Wiley, New York, 1962), p. 58.

${ }^{29}$ J. N. Smith and W. L. Fite, J. Chem. Phys. 37, 898 (1962).

${ }^{30}$ Landolt-Börnstein, 6th ed., Vol. II, Part 5b, Transportphänomene II, Kinetik, Homogene Gasgleichgewichte, edited by K. Schäfer (Springer, Berlin, 1968), Chap. 26.

${ }^{31}$ P. W. Atkins, Physical Chemistry (Oxford University Press, Oxford, 1994), Chap. 9.2.

${ }^{32}$ I. Barin, Thermochemical Data of Pure Substances (VCH, Weinheim, 1995).

${ }^{33}$ W. Steckelmacher, Rep. Prog. Phys. 49, 1083 (1986).

${ }^{34}$ In Eq. (2) of our previous paper (see Ref. 22), the second factor should read $U(\beta)$ instead of $U(\vartheta)$.

${ }^{35}$ P. Zugenmaier, Z. Angew. Phys. 20, 184 (1966), Eq. (5). 